\title{
COMPLICATED CASE OF RHEUMATIC HEART DISEASE; UNEXPLORED FOR YEARS
}

\author{
${ }^{1}$ Arif Khan, M., ${ }^{2}$ Raj Kishore Pandey, ${ }^{1}$ Tarique Nadeem, ${ }^{1}$ Manoj Kumar Sethi, \\ ${ }^{1}$ Sumit Sharma, ${ }^{1}$ Krishna Murti, ${ }^{1}$ Ashok Gupta and ${ }^{3}$ Krishna Pandey \\ ${ }^{1}$ Department of Pharmacy Practice, National Institute of Pharmaceutical Education and Research, Hajipur, India \\ ${ }^{2}$ Department of Biotechnology, National Institute of Pharmaceutical Education and Research, Hajipur, India \\ ${ }^{3}$ Department of Clinical Medicine, Rajendra Memorial Research Institute of Medical Sciences, Patna, India
}

Received 2013-12-24; Revised 2013-12-31; Accepted 2014-01-23

\begin{abstract}
Rheumatic heart disease is a one of the very common heart problem commonly prevalent among the children of developing countries, may occur in adults in their fourties also, if undiagnosed in early ages, similar in symptoms to 'rheumatism' but quite difficult to diagnose or are often misdiagnosed. The main objective of this article is to make acquainted about the disease and its further consequences, since initially it appears to be just a simple general fever but may prove deadly if undiagnosed or misdiagnosed leading to severe heart valve damage and consequent complications. The methods involved in the case include Bilateral Femoral Embolectomy (BFE), fasciotomy and Balloon Mitral Valvotomy (BMV). Through this case study an attempt has been made by the authors to make the people especially from the medical and related field, well acquainted about this deadly, silent, heart disease and its consequent complications.
\end{abstract}

Keywords: Rheumatic Heart Disease, Mitral Stenosis, Rheumatic Fever, Case Study

\section{INTRODUCTION}

Rheumatic Heart Disease Rheumatic Heart Disease (RHD) is one of the most commonly acquired heart diseases among the children of many countries of the world, especially in the developing countries. In 2005, it was estimated globally that over 2.4 million children aged 5-14 years are affected with RHD and $79 \%$ of all RHD cases come from less developed countries (Carapetis et al., 2005). Estimates suggests that at least 15.6 million people currently affected by RHD, responsible for about 233,000 deaths annually. The poorly affected areas of the world are subSaharan Africa, south-central Asia, the Pacific and indigenous populations of Australia and New Zealand (Mendis et al., 2011). Rheumatic heart disease is a consequence of Acute Rheumatic Fever (ARF). The risk of RHD is greater with repeated episodes of ARF.
RHD can damage any part of the heart including the valves, the lining of the heart or the heart muscle, but more often damages the heart valves, especially those on the left side of the heart. RHD does not always produce symptoms, but when it does, symptoms may include; chest pain, palpitations, exertional breathlessness, paroxysmal nocturnal dyspnoea, swelling (oedema), fainting (syncope). Rheumatic heart disease most often remain undiagnosed in its early stages because of the reason that it may or may not produce the symptoms. Also the symptoms which it produces are vague and may be confused with other conditions. It begins with a common throat infection caused by streptococcus bacteria which are 'rheumatogenic', mainly streptococcus pyogenes (Group 'A' Beta hemolytic streptococcal pharyngeal infection) which triggers off an abnormal immune reaction that damages heart valves.

Corresponding Author: Arif Khan, M., Department of Pharmacy Practice, National Institute of Pharmaceutical Education and Research, Hajipur, India Tel: +91-9470813689 
Acute rheumatic fever commonly appear in children aged between 6 and 15, with only around $20 \%$ of the attacks occurring in adults for the very first-time (Vinay et al., 2007) people can have recurrent episodes well into their forties also (NHFA, 2006). The illness is so named because of its similarity in symptoms with 'rheumatism'. Rheumatic fever is common worldwide with global estimates of 345,000 deaths upto 2010 which was 463,000 in 1990 (Lozano et al., 2012). The development rate of rheumatic fever in individuals with untreated streptococcal infection is estimated to be around 3\%. The incidence of recurrence with a subsequent untreated infection is about $50 \%$ which is substantially great (Carol, 2007).

\subsection{Case History}

A 26 year old young Indian was taken to a clinic because of severe pain in both of the lower limbs which occurred while playing table tennis. From there he was referred by the physician to a heart hospital for correct diagnosis and management.

\subsection{Case Presentation}

\subsubsection{Thromboembolic Obstruction of Aortic Bifurcation}

The patient was admitted to the heart hospital on 12 June 2013 with chief complaints of pain and discolouration of acute onset in both the threatened lower limbs. He was normotensive and non-diabetic. He underwent through investigations like Prothrombin Test/International Normalised Ratio (PT/INR) test and Echo test for serving the purpose of clinical diagnosis. PT with INR was found to be raised (1.81) and Color Doppler 2D Echo test revealed Rheumatic Heart Disease (RHD) and Severe Mitral Stenosis (MS).

The patient underwent Peripheral Angiography on 12 June 2013 (day of admission) which revealed obstruction at the aortic bifurcation with poor distal flow. On the same very day, he underwent through Bilateral Femoral Embolectomy (BFE) (emergency surgical procedure for removal of emboli impeding or blocking the blood circulation), with removal of large amount of blood clots. Since there was a massive swelling in the right calf, thus Fasciotomy (right leg) as well was done. There were no intraoperative and postoperative complications. After Embolectomy limb became warm, returned distal pulses. Wound healing was well with no any sign of infection.

\subsection{Admitting Diagnosis}

The final diagnosis which was made was; Thromboembolic obstruction of aortic bifurcation, Rheumatic heart disease, severe Mitral Stenosis, moderate pulmonary arterial hypertension with normal left ventricular function.

\subsection{Medication Advise}

The patient stayed in the hospital for the next seven days and was adviced for the following medications; Linezolid $(600 \mathrm{mg})$, an Oxazolidinone antimicrobial agent for the treatment resistant gram-positive coccal and bacillary infections, Cefuroxime (500 mg), a second generation Cephalosporin antibiotic, Warfarin ( $5 \mathrm{mg}$ ), a coumarin derivative oral anticoagulant, a combination of Furosemide $40 \mathrm{mg}$ (a high ceiling sulphamoyl derivative diuretic) and Amiloride $5 \mathrm{mg}$ (a potassium sparing diuretic), Digoxin (0.25 mg), a purified cardiac glycoside obtained from Digitalis lanata, a food supplement containing vitamin D3, B complex and Ginkgo biloba (Platelet Activating Factor antagonist), Esomeprazole (20 mg), a proton pump inhibitor, Paracetamol (650 mg) (acetaminophen), a Non Steroidal Anti-Inflammatory Drug (NSAID), Benzathine Penicillin-G (12 lac units I.M every 21 days). PT with INR was repeated every four hours till the day of discharge with dressing of wound changed every alternate day. He was discharged from the hospital on 19th of June 2013 with advice of PT/INR test to be done after three days. PT/INR was done on 22 June 2013 and was found to be slightly raised (1.94). He was adviced for surgeon consultation every week with reports of PT/INR test. On 01 July 2013 PT/INR was done and was found to be normal (2.59).

\subsection{Resurfacing of Fasciotomy Wound}

On 01 July 2013 the patient was referred to a plastic and cosmetic surgery clinic for resurfacing of fasciotomy wound where surgeon planned for debridement along with Split Skin Graft (SSG). Next day, serological and haematological investigations were done to proceed for the surgery. The serological tests included test for Australia Antigen (negative), HIV (Carapetis et al., 2005), (negative), HIV (Mendis et al., 2011), (negative). The haematological tests included investigation of level and was found to be $12.5 \mathrm{~g} \mathrm{dL}^{-1}(86.25 \%)$. On the same day lower intensity local anaesthesia, thorough wound scrapping and SSG was done. 


\subsection{Rheumatic Heart Disease and Severe Mitral Stenosis}

On 21 July 2013, the patient was again admitted in the same heart hospital for the purpose of Balloon Mitral Valvotomy (BMV) as recommended by the cardiac surgeon after operating for Bilateral Femoral Embolectomy with removal of blood clots and Fasciotomy of right leg. He was admitted with chief complaints and brief history of being normotensive and non diabetic, having known case of Rheumatic Heart Disease, severe mitral stenosis, mild mitral regurgitation, moderate pulmonary arterial hypertension, normal sinus rhythm, no clots, Femoral Embolectomy and complaints of dyspnea on effort.

\subsection{Investigations}

Physical examination revealed; Heart Rate-70/min, Blood Pressure 110/70 mmhg, chest B/L clear, heart sound 1 increased, Opening Snap present (OS+) (Loud S1 and OS indicate leaflet pliability and tells that the valve is not fibrosed or calcified, do not predict severity) along with mild Mid Diastolic Murmer (MDM+) (best heard at apex). Loud sound 1, opening snap and mid diastolic murmer are the cardiovascular auscultations among the diagnostic features of mitral stenosis. Chest X- Ray revealed Cardiothoracic Ratio (CTR) -55\% with no Pulmonary Venous Hypertension (PVH). CTR is a ratio between cardiac width and thoracic width, ratio of $>1: 2 \quad(50 \%)$ is considered to be abnormal. Electrocardiography revealed Normal Sinus Rhythm (NSR), Right Ventricular Hypertrophy (RVH) with Right Axis Deviation (RAD) confirmed the diagnosis along with assessment of severity. Echo test was done before and after BMV. Pre BMV, Mitral Valve Orifice Area (MVOA) was revealed to be $0.8 \mathrm{~cm}^{2}$ (normal area is 4-6 $\mathrm{cm}^{2}$ ), mild Mitral Regurgitation (MR), mean pressure gradient was $16 \mathrm{mmHg}$ (mean pressure gradient is usually higher than $10 \mathrm{mmHg}$ in severe stenosis) and Right Ventricular Systolic pressure was $80 \mathrm{mmHg}$ (RVSP $\leq 35 \mathrm{mmHg}$ is considered to be normal while RVSP $>40 \mathrm{mmHg}$ indicates mild pulmonary hypertension). Post BMV, MVOA was found to be $1.7 \mathrm{~cm}^{2}$, mild $\mathrm{MR}$, mean pressure gradient was $6 \mathrm{mmHg}$ with RVSP $50 \mathrm{mmHg}$.

\subsection{Hospital Course}

After mandatory investigations, Ballon Mitral Valvotomy was done successfully on 22 July 2013 via right femoral approach. Single uncomplicated transeptal puncture was done using $28 \mathrm{~mm}$ Accura balloon with single inflation done $(26 \times 1)$. MDM was reduced. Patient remained haemodynamically stable during operation. Left Arterial (LA) Pressure reduced to $6 \mathrm{mmHg}$ from 20 $\mathrm{mmHg}$. Hospital Stay was uneventful.

\subsection{Medication Advise}

The patient was discharged from the hospital on 23 July 2013 in stable condition with advice for the following given medications; Benzathine Penicillin G (12 Lac units), deep I.M, Digoxin $(0.25 \mathrm{mg})$, a combination of Furosemide and Amiloride (40 $\mathrm{mg}$ and $5 \mathrm{mg}$ respectively), Nicoumalone (3 $\mathrm{mg}$ ) with rest and review after one week. On 31 July 2013 $\mathrm{PT} / \mathrm{INR}$ was done and was found to be normal along with normal BP and pulse rate. Metoprolol $(50 \mathrm{mg})$ was added with the above medications.

\section{DISCUSSION}

Mitral Stenosis (MS) refers to the condition of pathological narrowing of the mitral valve orifice, resulting in impedance of filling of the left ventricle in diastole. The most commonest cause is Rheumatic Heart Disease, the rare causes include calcification of mitral annulus, Congenital, Lutembacher's syndrome, Infective endocarditis, Endomyocardial fibrosis. Hurler's syndrome, Rheumatoid Arthritis and Methysurgide. Approximately about $25 \%$ of population with RHD has pure MS while additional $40 \%$ population has MS along with MR (Dare, 1993; Waller et al., 1994). The most common presenting symptom in patients with mitral stenosis is progressive exertional dyspnea, along with fatigue, atrial arrhythmias, hemoptysis, palpitation, thromboembolism, angina-like chest pain, or even right-sided heart failure. Here the patient was admitted with complaints of fatigue, where he was diagnosed with thromboembolism along with rheumatic heart disease. A study conducted by Vijayalakshmi et al. (2010) showed that over expression of CD44 protein (receptor for the ligand hyaluronic acid which causes inflammation in the heart valves) is a critical finding in patients with stenosis and regurgitation. (Vijayalakshmi et al., 2010) mitral stenosis has a slow and stable course in the early years followed by a progressive acceleration later in life (Wood, 1954). In India, critical MS may be found in children as young as 6 to 12 years of age. In this case MS is diagnosed at the age of 26 years which is quite a late age for diagnosis and the case proved to be a hidden entity for a longer duration. Other complications might have occurred as a consequence of this hidden duration and the case would have become more complicated than it actually is. The patient 
underwent thromboembolectomy for the removal of blood clots in large numbers, producing impedence to the blood circulation and the circulation was almost blocked in the lower limb due to which the leg became threatened and would have produced more complications and if this surgical intervention could not have done for next few hours, the chances for infections could have been more pronounced, threatening the leg and rendering the case more complicated. In the asymptomatic or minimally symptomatic patient, survival is greater than $80 \%$ at 10 years, with $60 \%$ of patients having no or very lesser progression of symptoms (Olesen, 1962; Selzer and Cohn, 1972). Normal Mitral Valve Orifice Area (MVOA) is 4.0$6.0 \mathrm{~cm}^{2}$. MVOA of less than $1.0 \mathrm{~cm}^{2}$ is graded to be as severe MS, MVOA between $1.5-1.0 \mathrm{~cm}^{2}$ is graded as moderate MS whereas MVOA lying between $1.5-2.5 \mathrm{~cm}^{2}$ is considered to be mild stenosis. It was a case of severe MS having MVOA of $0.8 \mathrm{~cm}^{2}$ before but after BMV it became mild stenosis having MVOA of $1.7 \mathrm{~cm}^{2}$. When MVOA goes below $1 \mathrm{~cm}^{2}$, there will be an increase in left arterial pressure and valve causes an impediment to the flow of blood into left ventricle, creating a pressure gradient across mitral valve.

Available treatment options include 1. Medical Therapy 2. Balloon Mitral Valvotomy (BMV) 3. Surgical Valvotomy (Closed Mitral Valvotomy (CMV) or Open Mitral Valvotomy (OMV)/Mitral Valve Replacement MVR]. In general, patients having MS should undergo antibiotic prophylaxis for prevention from procedures known to cause bacteremia. Diuretics are quite useful for treating milder symptoms of hypertension. Beta blockers attenuate the increase in heart rate during exertion and or physical labour. Digoxin has no role in patients with MS. Immediate control can be accomplished by administration of $\mathrm{B}$ blockers or calcium channel blockers (diltiazem and verapamil derivatives). Consequently, all the patients require warfarin anticoagulant therapy with a target International Normalized Ratio (PT/INR) of 2.0 to 3.0.

Baloon Mitral Valvotomy is the preferred treatment for mitral valve stenosis, because it does not require any open heart surgery., BMV has become the treatment of choice for patients with symptomatic MS in the past two decades after its introduction in 1984 by Inoue et al. (1984) and Mendis et al. (2011). Transseptal technique is the most common technique used to perform BMV. Accura or Inoue balloon advanced over the wire (catheter) with a transseptal puncture inflated after being positioned across the mitral valve. Stepwise dilatation with gradual increase in the size of balloon is performed which is monitored by the echocardiographic and hemodynamic assessment. BMV is considered to be successful if post BMV MVOA becomes $>1.5 \mathrm{~cm}^{2}$ or if there is $50 \%$ increase in MVOA compared to pre BMV valve area. Here in this case post BMV MVOA became $1.7 \mathrm{~cm}^{2}$ from $0.8 \mathrm{~cm}^{2}$, more than $50 \%$ increase, hence this intervention proved to be successful. Overall, 80 to $95 \%$ of population treated with a balloon valvotomy have a successful outcome (Bonow, 2006).

\section{CONCLUSION}

Around six months prior to the diagnosis of RHD, the patient suffered from cough and throat infection and for that he consulted a local physician by whom he was informed that he is having some kind of defect in heart valve and was adviced to go for ECG and Echo test. But the advice was not taken seriously since nobody is going for Echo for just having simple cold and cough, also having a defect in heart valve or something itself creates tension in the mind of patient and thus becomes a matter of great concern. That throat infection may be attributable to Streptococcus bacteria which is the main cause of rheumatic fever but since many a times symptoms are not necessarily produced, therefore it becomes very difficult to diagnose, especially rheumatic fever. In this case it may be a cause that remained undiagnosed or poorly diagnosed at the very first time, we cannot say that it was completely undiagnosed because the physician for sure noted some defect in heart valve, although the cause was not exactly clear but the probable consequence was clear. So it must be stated that it was the excellent diagnosis of that physician which was not taken seriously, consequently producing complications in the case and might have become more complicated in just a matter of little time. The authors feel that there is a need to emphasize on the fact that the physician's advices should not be taken very lightly and the patients should not make their own assumptions as, for them it may produce complications and may prove to be a matter of life and death.

\section{LIMITATIONS}

The limitation which is supposedly felt here to be significant in this case of rheumatic heart disease is most importantly the diagnosis or say the misdiagnosis of the rheumatic heart disease which generally presents with the common symptoms as that of fever or rheumatic fever like here in this case, progressing to 
mitral stenosis and mitral regurgitation very silently and steadily, thus concluding that the limitation lies in the clinical diagnosis of the case which requires a deep insight of the condition, for which an attempt has been made here in this case.

\section{REFERENCES}

Bonow, R.O., 2006. ACC/AHA 2006 guidelines for the management of patients with valvular heart disease. A report of the American College of Cardiology/American Heart Association Task Force on Practice Guidelines (Writing Committee to Revise the 1998 Guidelines for the Management of Patients with Valvular Heart Disease). Circulation, 114: e84-e231. DOI: 10.1161/CIRCULATIONAHA.106.176857

Carapetis, J.R., A.C. Steer, Mulholl and M.E.K. Weber, 2005. The global burden of group a streptococcal diseases. Lancet Infect Dis., 5: 685-694. DOI: 10.1016/S1473-3099(05)70267-X

Carol, P., 2007. Essentials of Pathophysiology: Concepts of Altered Health States. 1st Edn., Lippincott Williams and Wilkins, Philadelphia, ISBN-10: 0781770874 , pp: 1147.

Dare, A., 1993. Evaluation of surgically excised mitral valves: Revised recommendations based on changing operative procedures in the 1990s. Hum. Pathol., 24: 1286-1293. PMID: 8276375

Inoue, K., T. Owaki, T. Nakamura, F. Kitamura and N. Miyamoto, 1984. Clinical application of transvenous mitral commissurotomy by a new balloon catheter. J. Thorac. Cardiovasc. Surg., 87: 394-402. PMID: 6700245
Lozano, R., M. Naghavi, K. Foreman, S. Lim and K. Shibuya et al., 2012. Global and regional mortality from 235 causes of death for 20 age groups in 1990 and 2010: A systematic analysis for the Global Burden of Disease Study 2010. Lancet, 380: 2095-2128. DOI: 10.1016/S0140-6736(12)61728-0, PMID: 23245604

Mendis, S., P. Puska and B. Norrving, 2011. Global Atlas on Cardiovascular Disease Prevention and Control. World Health Organization, Geneva, ISBN10: $9241564377, \mathrm{pp}: 155$.

NHFA, 2006. Diagnosis and management of acute rheumatic fever and rheumatic heart disease in Australia: An evidence based review. National Heart Foundation of Australia.

Olesen, K.H., 1962. The natural history of 271 patients with mitral stenosis under medical treatment. Br. Heart J., 24: 349-357. DOI: 10.1136/hrt.24.3.349

Selzer, A. and K.E. Cohn, 1972. Natural history of mitral stenosis a review. Circulation, 45: 878-890. DOI: 10.1161/01.CIR.45.4.878

Vinay, K., K.A. Abul, N. Fausto and R. Mitchell, 2007. Robbins Basic Pathology. 8th Edn., Elsevier Health Sciences, Philadelphia, ISBN-10: 1437700667, pp: 960.

Vijayalakshmi, R., S. Nayar and K.M. Cherian, 2010. Expression of the CD44 protein in the heart valves affected with rheumatic heart disease. Am. J. Infect. Dis., 6: 122-124. DOI: 10.3844/ajidsp.2010.122.124

Waller, B., J. Howard and S. Fess, 1994. Pathology of mitral valve stenosis and pure mitral regurgitation-Part I. Clin. Cardiol., 17: 330-336. PMID: 8070151

Wood, P., 1954. An appreciation of mitral stenosis, clinical features. Br. Med. J., 4870: 1051-1063. DOI: $10.1136 / \mathrm{bmj} .1 .4870 .1051$ 